\title{
Efficacy and safety of bevacizumab combined with chemotherapy in symptomatic brain metastases from lung adenocarcinoma: a retrospective analysis
}

\author{
Qiong Zhan ${ }^{1 \#}$, Feng Miao ${ }^{2 \#}$, Ruofan Huang ${ }^{1}$, Xinli Zhou ${ }^{1}$, Mengxi Ge ${ }^{2}$, Xiaohua Liang ${ }^{1}$ \\ ${ }^{1}$ Department of Oncology, Huashan Hospital, Fudan University, Shanghai 200040, China; ${ }^{2}$ Department of Thoracic Surgery, Huashan Hospital \\ North, Fudan University, Shanghai 200040, China \\ Contributions: (I) Conception and design: Q Zhan, F Miao, M Ge, X Liang; (II) Administrative support: X Liang, M Ge; (III) Provision of study \\ materials or patients: X Liang, M Ge; (IV) Collection and assembly of data: Q Zhan, F Miao, R Huang, X Zhou; (V) Data analysis and interpretation: \\ Q Zhan, F Miao, R Huang, X Zhou; (VI) Manuscript writing: All authors; (VII) Final approval of manuscript: All authors. \\ \#These authors contributed equally to this work. \\ Correspondence to: Xiaohua Liang; Mengxi Ge. Department of Oncology, Huashan Hospital, Fudan University, Shanghai 200040, China. \\ Email: xhliang66@sina.com; gmxily@126.com.
}

Background: Currently, the treatment of symptomatic brain metastases from lung adenocarcinoma has remained difficult. Bevacizumab combined with chemotherapy is one of the standard treatments of lung adenocarcinoma. This study was designed to investigate the efficacy and safety of bevacizumab combined with chemotherapy in symptomatic brain metastases from lung adenocarcinoma that are not suitable for local treatments, and to explore the predictive value of baseline serum vascular endothelial growth factor (VEGF) for the treatment.

Methods: We retrospectively reviewed 14 consecutive patients, between Jan 2015 and Jul 2017, with brain metastases from lung adenocarcinoma who received bevacizumab and chemotherapy to determine efficacy and toxicity. Kaplan-Meier method was used to estimate survival curves, and univariate and multivariate analyses were performed by Cox proportional hazard model. The primary endpoints were objective response rate (ORR) and intracranial ORR (iORR). The secondary endpoints were progression-free survival (PFS), intracranial PFS (iPFS), overall survival (OS) and disease control rate (DCR).

Results: The efficacy of 12 patient was evaluated. Overall ORR was 25\% (3/12) and the iORR of brain lesions was $33.3 \%$ (4/12). DCR was $75 \%$ (9/12). The median OS was 18.3 months, the median PFS was 6.7 months, and the median iPFS was 12 months. After 2 cycles of bevacizumab, 10 patients showed improved symptoms of central nervous system (CNS), and the symptom control rate was 83.3\% (10/12). Head MRI showed that edema in the brain was greatly reduced in 6 patients, resulting in the lessened usage of dexamethasone. iPFS was significantly shorter in high VEGF group (3.6 vs. $8.0 \mathrm{~m}, \mathrm{P}=0.02$ ), and multivariate analysis showed a significant correlation between iPFS and serum baseline VEGF level $(\mathrm{P}=0.023)$. The most commonly adverse events of bevacizumab included leukopenia [5 (35.7\%)], fatigue [3 (21.4\%)], thrombocytopenia [3 (21.4\%)], anemia [2 (14.3\%)], which were mostly degree I and II.

Conclusions: This study showed bevacizumab combined with chemotherapy could effectively control intracranial lesions, relieve symptoms, and improve the quality of life and survival of patients with brain metastases from lung adenocarcinoma. Serum baseline VEGF may be a predictor of efficacy of bevacizumab plus chemotherapy in the treatment of brain metastases from lung adenocarcinoma.

Keywords: Symptomatic brain metastases; lung adenocarcinoma; bevacizumab plus chemotherapy; vascular endothelial growth factor (VEGF)

Submitted Aug 01, 2019. Accepted for publication Oct 10, 2019.

doi: $10.21037 /$ jtd.2019.10.49

View this article at: http://dx.doi.org/10.21037/jtd.2019.10.49

(C) Journal of Thoracic Disease. All rights reserved. 


\section{Introduction}

The brain is one of the most common metastatic sites of non-small cell lung cancer (NSCLC), occurring about 50\% of lung adenocarcinoma during the course of the disease. NSCLC patients with brain metastases, which are one of the major causes of treatment failure or even deaths, have a poor prognosis with a median overall survival (OS) of approximately $1-2$ months $(1,2)$. The usage of tyrosine kinase inhibitor (TKI), such as gefitinib, osimertinib, crizotinib and ceritinib, has improved the intracranial response rate and survival of driver-gene-mutant patients with brain metastases from lung adenocarcinoma (3). However, for patients without driver mutations or that resistant to TKI, especially with symptoms of central nervous system (CNS), there are no more effective treatment beside chemotherapy or radiotherapy, which efficacy remains dissatisfactory.

Bevacizumab is a recombinant humanized monoclonal lgG1 antibody, which has anti-tumor activity by inhibiting vascular endothelial growth factor (VEGF). Bevacizumab combination with chemotherapy could significantly improve survival of nonsquamous NSCLC, so the combination therapy was approved for the treatment of NSCLC as the first-line treatment (4-6). Bevacizumab provides a new choice for treatment of NSCLC patients with brain metastases (7-9). A phase II clinical trial assessing bevacizumab in nonsquamous NSCLC with asymptomatic brain metastases showed a median progression-free survival (mPFS) of 6.7 months and a median overall survival (mOS) of 16.0 months (10). Bennouna et al. (11) reported that nonsquamous NSCLC with or without brain metastases treated by bevacizumab combination with chemotherapy had similar mPFS (6.5 vs. 6.9 months, $\mathrm{P}=0.54)$ and $\mathrm{mOS}$ (14.5 vs. 12.5 months, $\mathrm{P}=0.33$ ).

Patients with brain metastases were excluded in the early phase clinical trials of bevacizumab. Although several recent retrospective studies and small clinical trials included patients with brain metastases, most of subjects were asymptomatic (8-11). Nevertheless, the NSCLC patients with symptomatic brain metastases are more common in the real world. Neurosurgical resection, intracranial stereotactic radiosurgery (SRS), and whole brain irradiation (WBRT) are main treatment options for patients with symptomatic brain metastases, whereas for patients who are ineligible to receive surgery or refuse local therapy because of radiationinduced neurotoxicity, drug therapy plays a key role in disease control and symptom improvement. Cerebral edema could be controlled by corticosteroids, but long-term use of corticosteroids may result in serious adverse events such as Cushing syndrome and reduced quality of life. Previous clinical studies demonstrated that bevacizumabbased treatment could effectively improve symptoms of patients with glioblastoma, allowing lessened usage of corticosteroids $(12,13)$. Furthermore, a case reports showed that bevacizumab led to alleviation of cerebral edema and reduced dosage of corticosteroids in brain-metastatic breast cancer (14).

We conducted the retrospective study to investigate the efficacy and safety of bevacizumab combined with chemotherapy in symptomatic brain metastases from lung adenocarcinoma, and to explore the predictive value of baseline serum VEGF for the treatment.

\section{Methods}

\section{Patient characteristics}

In this retrospective study, we enrolled consecutive patients with symptomatic brain metastases from lung adenocarcinoma at Department of Oncology, Huashan Hospital (Shanghai, China), from January 2015 to July 2017. We included patients aged at least 18 years; a histologically confirmed diagnosis of lung adenocarcinoma; brain metastases or leptomeningeal metastases confirmed by CT or MRI, and/or histologically confirmed diagnosis of brain metastases or cerebrospinal fluid (CSF) cytology positive; no EGFR-sensitizing mutation or ALK translocations, or progression after an appropriate TKI for those with a sensitizing EGFR mutation or ALK gene rearrangement; with symptoms of CNS, but without brain metastases crisis (15); ineligible for or refuse of local therapy (surgery or radiation) for intracranial lesion, or interval time between end of brain irradiation and beginning of the treatment with bevacizumab $>3$ months; clinical indication for chemotherapy, including normal peripheral hemogram, no abnormalities of heart, liver and kidney function, normal electrocardiograph, no severe hypertension (blood pressure $<150 / 100 \mathrm{mmHg}$ ) or hemorrhagic disease; no albuminuria (>2 g/24 h); and no unhealed wound. All study participants provided informed written consent. The protocol was approved by the Institutional Review Board Committee of Huashan hospital, Shanghai, China (No. KY2017-010). Participants were followed up every month and the last date of followup was June 30, 2018. 


\section{Treatment}

Participants received bevacizumab $7.5 \mathrm{mg} / \mathrm{kg}$ intravenously every 3 weeks. Double platinum chemotherapy was given to those with ECOG performance status of 0 or 1 . The chemotherapy agent for the patient with PS of 3 was pemetrexed. The two of patients with PS of 2 received pemetrexed plus cisplatin, and the other three received docetaxel and pemetrexed, respectively. The third generation chemotherapy agents which were previously effective or unused were chosen as the chemotherapy program. Chemotherapy agents were administered as follow: paclitaxel $135 \mathrm{mg} / \mathrm{m}^{2}$, intravenously; pemetrexed $500 \mathrm{mg} / \mathrm{m}^{2}$, intravenously; docetaxel $75 \mathrm{mg} / \mathrm{m}^{2}$, intravenously; cisplatin, $75 \mathrm{mg} / \mathrm{m}^{2}$, intravenously.

\section{Assessments}

Chest and abdomen CT and head MRI were performed every 6 weeks. The response to treatment was assessed according to RECIST (version 1.1) (16), including complete remission (CR), partial remission (PR), stable disease (SD) and progression disease (PD). Intracranial lesion was assessed according to RANO-BM (Response Assessment in Neuro-Oncology Brain Metastases) $(17,18)$, consisting of intracranial CR (iCR), intracranial PR (iPR), intracranial $\mathrm{SD}$ (iSD), and intracranial PD (iPD). PFS was defined as time between beginning of bevacizumab treatment and disease progression or death. Intracranial PFS (iPFS) was defined as time between beginning of bevacizumab treatment and intracranial disease progression or death. OS was defined as time between beginning of bevacizumab treatment and death or last follow-up. All adverse events were graded according to the National Cancer Institute Common Terminology Criteria for Adverse Events, version 4.0 (19).

The primary endpoints were objective response rate (ORR) and intracranial ORR (iORR). The secondary endpoints were PFS, iPFS, OS and disease control rate (DCR).

\section{Detection of baseline serum VEGF}

Peripheral blood $(5 \mathrm{~mL})$ was collected from every participant before bevacizumab treatment. Blood samples were centrifuged at $4,000 \mathrm{r} / \mathrm{min}$ for $10 \mathrm{~min}$, and the serum samples were stored at $-20^{\circ} \mathrm{C}$. The level of VEGF was evaluated using the enzyme-linked immunosorbent assay (ELISA) (human VEGF ELISA Kit, BioVision) and detected utilizing fully automated electrochemiluminescence (Elecsys 2010, Roche).

\section{Statistical analysis}

All statistical analyses were conducted using Statistical Package for the Social Sciences for Windows, software version 18.0 (IBM Inc., Armonk, NY, USA). Continuous data are presented as the mean \pm standard deviation. PFS and OS curves were analyzed using the KaplanMeier method, with $\mathrm{P}$ value determined by a log-rank test. Hazard ratios (HRs) and $95.0 \%$ confidence intervals (CIs) were calculated. Univariate and multivariate analyses were performed by Cox proportional hazard model. In multivariate analyses, the clinicopathological characteristics with $\mathrm{P}<0.1$ in univariate analysis were included to identify independent prognostic factors. A two-sided $\mathrm{P}<0.05$ was considered statistically significant.

\section{Results}

\section{Patients characteristics}

From January 2015 to July 2017, a total of 14 patients with symptomatic brain metastases from lung adenocarcinoma were enrolled. Detailed clinicopathological data the cohort is shown in Table 1 and Table S1. In brief, 7 patients (50\%) had metastasis lesion besides brain, and $50 \%$ patients with drive genes mutation (EGFR or ALK), who received fistline treatment with EGFR-TKI or ALK-TKI, such as erlotinib, gefitinib, osimertinib, ecotinib, or crizotinib. Eleven patients (78.6\%) received pemetrexed plus cisplatin or paclitaxel plus cisplatin as chemotherapy program. The median courses of bevacizumab treatment cycles was 2.5 (range, 1-15).

\section{Efficacy}

Up to the last follow-up, all participants received a total of 102 cycles of bevacizumab treatment, and the median number of bevacizumab treatment cycles was 6 (range, 1-19). A proportion of $35.7 \%$ patients (5/14) received more than 10 cycles of bevacizumab, and two patients were still receiving bevacizumab at the time of data cutoff. Two patients received only one dose of bevacizumab because of adverse events or economic problems, which were excluded from efficacy analysis.

At the time of analysis, all of 12 patients had discontinued 
Table 1 Clinical characteristics

\begin{tabular}{|c|c|}
\hline Clinical characters & No. of patients $(n=14)$ \\
\hline Median age, years [range] & $60.5[42-71]$ \\
\hline \multicolumn{2}{|l|}{ Sex } \\
\hline Male & 10 \\
\hline Female & 4 \\
\hline \multicolumn{2}{|l|}{ ECOG PS } \\
\hline 1 & 8 \\
\hline 2 & 5 \\
\hline 3 & 1 \\
\hline \multicolumn{2}{|l|}{ Metastases outside the CNS } \\
\hline Yes & 7 \\
\hline No & 7 \\
\hline \multicolumn{2}{|l|}{ Driver-oncogene status } \\
\hline EGFR & 7 \\
\hline ALK fusion & 1 \\
\hline None & 6 \\
\hline \multicolumn{2}{|l|}{ Chemotherapy } \\
\hline Pemetrexed plus cisplatin & 8 \\
\hline Paclitaxel plus cisplatin & 3 \\
\hline Pemetrexed & 1 \\
\hline Docetaxel & 2 \\
\hline \multicolumn{2}{|l|}{ Baseline VEGF } \\
\hline High VEGF group & 6 \\
\hline Control group & 6 \\
\hline N/A & 2 \\
\hline
\end{tabular}

VEGF, vascular endothelial growth factor; CNS, central nervous system; EGFR, epidermal growth factor receptor; ALK, anaplastic lymphoma kinase.

treatment (Figure 1). Assessments of best response according to RECIST 1.1 were PR in 3 patients, SD in 6 patients, and PD in 3 patients (Table 2). ORR was $25 \%(3 / 12)$ and DCR was $75 \%$ (9/12). Assessments of intracranial lesion according to RANO-BM were iPR in 4 patients, iSD in 5 patients and iPD in 3 patients. iORR of brain lesions was $33.3 \%(4 / 12)$ and iDCR was $75 \%(9 / 12)$. The median OS was 18.3 months (95\% CI, 14.1-22.4 m), the median PFS was 6.7 months ( $95 \% \mathrm{CI}, 4.5-11.65 \mathrm{~m}$ ), and the median iPFS was 12 months (95\% CI, 6.6-16.3 m, Figure 2).
Before treatment of bevacizumab combined with chemotherapy, the main symptoms of CNS included limb headache $(41.7 \%)$, weakness $(33.3 \%)$, dizziness $(25.0 \%)$ and dysphasia (16.7\%), and four patients needed mannitol \pm dexamethasone to control these symptoms. After two cycles of bevacizumab treatment, improved symptoms of CNS were observed in ten patients (Table 2), resulting in a symptom control rate of $83.3 \%(10 / 12)$. The median time to improve the CNS symptoms from beginning of bevacizumab treatment was 270 days (range, $30-570$ days). Head MRI showed that edema in the brain was greatly reduced in 6 patients, resulting in the lessened usage of dexamethasone (Figure 3).

\section{Adverse events}

Adverse events were assessed in all of 14 patients (Table 3). Most treatment-related adverse events were grade 1 or 2, including leukopenia [5 (35.7\%)], fatigue [3 (21.4\%)], thrombocytopenia [3 (21.4\%)], anemia [2 (14.3\%)], etc. Grade 3 leukopenia occurred in 2 patients (14.3\%), who recovered following treatment with granulocyte-colony stimulating factor (G-CSF). One patient (7.1\%) had grade 1 hypertension and blood pressure returned to normal after treatment with nifedipine sustained-release tablets. One patient, who had albuminuria $(<2 \mathrm{~g} / 24 \mathrm{~h})$ before treatment, discontinued bevacizumab treatment because of increasing albuminuria ( $>2 \mathrm{~g} / 24 \mathrm{~h}$ ) after one cycle of bevacizumab. No grade 4 or 5 occurred. No hemoptysis, intracerebral hemorrhage or thrombotic diseases occurred in the patients.

\section{The baseline serum VGFR and the efficacy of bevacizumab combined with chemotherapy}

In efficacy analysis set of 12 patients, the median level of baseline serum VEGF was $61.3 \mathrm{pg} / \mathrm{mL}(3.2-281.9 \mathrm{pg} / \mathrm{mL})$. We defined the patients with higher VEGF than the median level of VEGF as high VEGF group, and the other patients as control group. Survival curve showed that iPFS was significantly shorter in high VEGF group $(3.6 v s .8 .0 \mathrm{~m}$, $\mathrm{P}=0.02$, Figure 4). Compared with control group, high VEGF was also associated with shorter PFS (5.8 vs. $12.1 \mathrm{~m}$ ) and OS (12.8 vs. $18.4 \mathrm{~m}$ ), but neither had significantly difference ( $\mathrm{P}=0.4$ and 0.08 , respectively, Figure 4$)$.

Univariate analyses showed that age, ECOG performance status, and baseline VEGF were associated with iPFS ( $\mathrm{P}=0.005$, Table 4). In multivariate logistic 


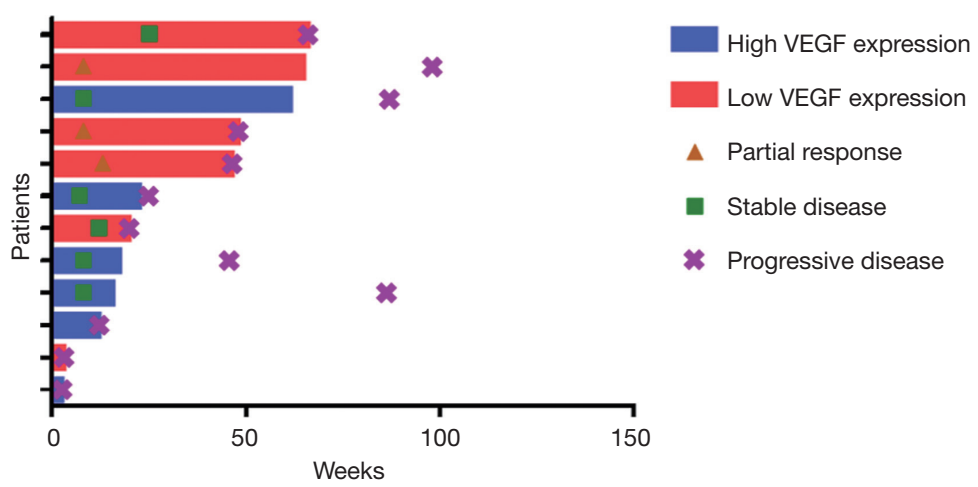

Figure 1 Treatment exposure and response duration. The length of each bar represents the treatment exposure of bevacizumab.

Table 2 Efficacy of bevacizumab

\begin{tabular}{lc}
\hline Response evaluation $(\mathrm{n}=12)$ & No. $(\%$ or $95 \% \mathrm{Cl})$ \\
\hline Response according to RECIST v1.1 & $3(25.0)$ \\
PR & $6(50.0)$ \\
SD & $3(25.0)$ \\
PD & \\
Intracranial response & $4(33.3)$ \\
iPR & $5(41.7)$ \\
iSD & $3(25.0)$ \\
iPD & \\
Symptom of CNS & 10 \\
Improved & 1 \\
No change & 1 \\
Increased & $6.7(4.5-11.65)$ \\
PFS (m) & $12(6.6-16.3)$ \\
iPFS (m) & $18.3(14.1-22.4)$ \\
OS (m) & \\
\hline Cl confidence interval; CNS, & \\
\hline
\end{tabular}

$\mathrm{Cl}$, confidence interval; CNS, central nervous system; m, months; $\mathrm{PR}$, partial response; SD, stable disease; PD, progression disease; PFS, progression-free survival; iPR, intracranial partial response; iSD, intracranial stable disease; iPD, intracranial progression disease; iPFS, intracranial progression-free survival; OS, overall survival.

regression including age, ECOG performance status, and baseline VEGF, the association between baseline VEGF and iPFS remained significant $(\mathrm{P}=0.023$, Table 5). These results indicated that baseline serum VEGF could serve as a promising biomarker to predict the efficacy of bevacizumab combined with chemotherapy in symptomatic brain metastases from lung adenocarcinoma.

\section{Discussion}

As an antiangiogenic agent, bevacizumab could make blood vessel normal, enhance vascular permeability and improve tumor microenvironment $(20,21)$. Fischer et al. (22) observed that microvascular density in tumors was decreased and vascular morphology was normalized after bevacizumab treatment. A preclinical study demonstrated that inhibiting VEGF could prevent brain metastases in mouse model (23). A retrospective analysis showed bevacizumab combining with chemotherapy significantly reduced the incidence of brain metastasis from lung compared with chemotherapy alone $(14 \%$ vs. $31 \%, \mathrm{P}<0.05)$ (24). On the other side, peritumoral edema often happens when incomplete vascular endothelium and increased vascular permeability cause fluid and serum protein to leak into nearby tissues. Increasing VEGF expression is associated with peritumoral edema, so anti-VEGF therapy could effectively control peritumoral edema and improve symptoms of CNS (25-27).

Unlike other studies, we enrolled the patients with symptomatic brain metastases, including some patients with worse ECOG performance status $(\geq 2,42.9 \%)$. Compared with previous studies of asymptomatic brain metastases, we observed similar OS and PFS. What's more, intracranial efficacy was remarkable, including high iORR and iDCR (33.3\% and $75 \%$, respectively). More than $90 \%$ patients obtained improved symptoms of CNS, resulting in lessened usage of dexamethasone and improved quality of life. Our results suggest that bevacizumab combination with chemotherapy could offer a choice to the patients with 

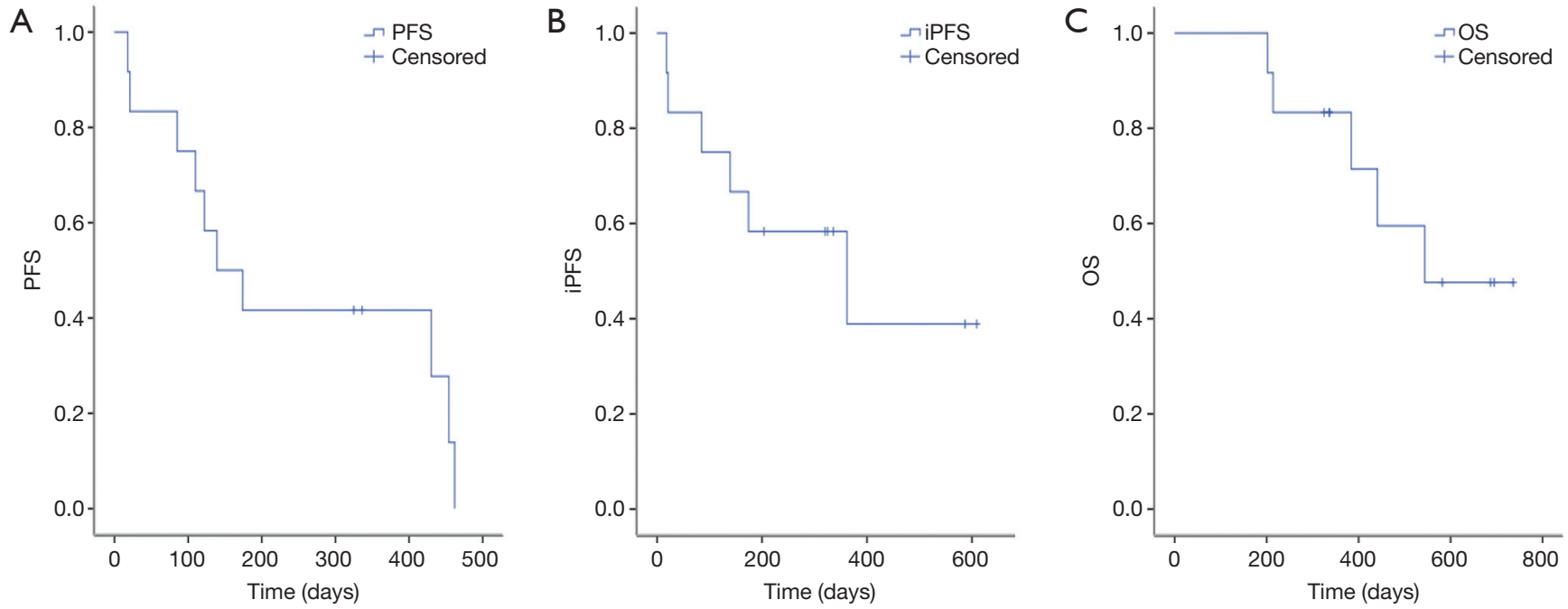

Figure 2 Kaplan-Meier estimates of progression-free survival (A), intracranial progression-free survival (B) and overall survival (C).
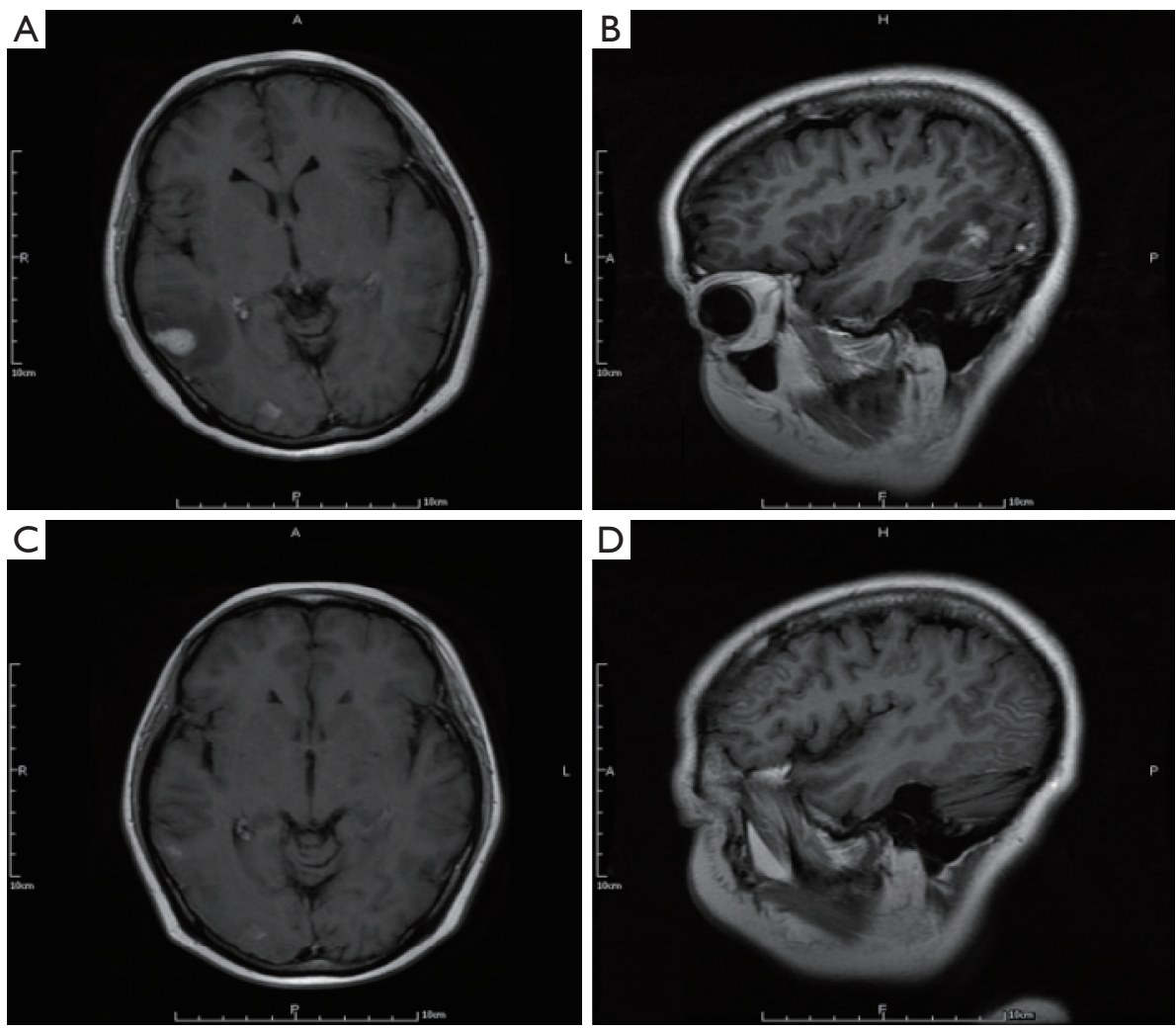

Figure 3 The brain MRI of a lung adenocarcinoma patient with brain metastasis before (A,B) and after (C,D) 2 cycles of bevacizumab.

symptomatic brain metastases who are ineligible to local therapy, even for the patients who are eligible to receive local therapy, bevacizumab combination therapy could improve symptoms and postpone local therapy.
Several studies also revealed bevacizumab combination with chemotherapy was a safe treatment for patients with brain metastasis from lung, with low incidence of intracerebral hemorrhage and other adverse events. 
Table 3 Treatment-related adverse events

\begin{tabular}{lcc}
\hline Adverse events, $\mathrm{n}=14$ & All grades, No. (\%) & Grade $3^{\mathrm{a}}$, No. (\%) \\
\hline Leukopenia & $7(50.0)$ & 0 \\
Fatigue & $3(21.4)$ & 0 \\
Thrombocytopenia & $3(21.4)$ & 0 \\
Anemia & $2(14.3)$ & 0 \\
DVT & $1(7.1)$ & 0 \\
Hypertension & $1(7.1)$ & $1(7.1)$ \\
Albuminuria & $1(7.1)$ & 0 \\
Epistaxis & $1(7.1)$ & 0 \\
Creatinine increased & $1(7.1)$ & 0 \\
ALT increased & $1(7.1)$ &
\end{tabular}

${ }^{\mathrm{a}}$, there were no grade 4 or 5 treatment-related adverse events. ALT, alanine aminotransferase; DVT, deep vein thrombosis.
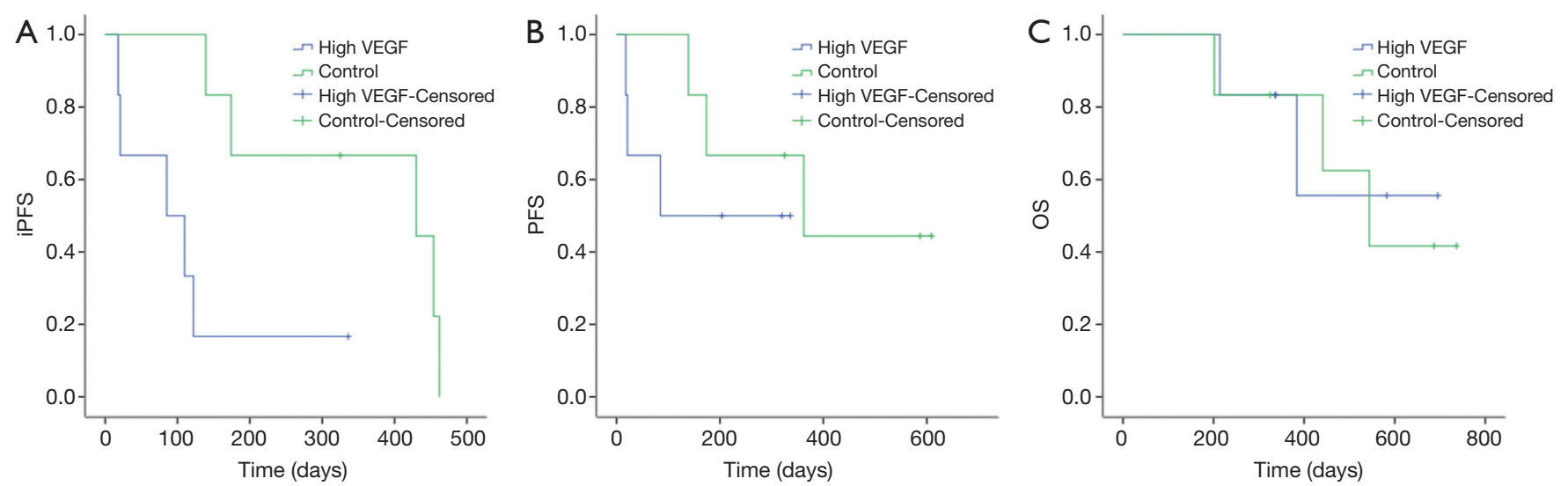

Figure 4 The baseline VEGF level and efficacy of bevacizumab combining with chemotherapy. (A) iPFS (high VEGF group vs. control group: 3.6 vs. $8.0 \mathrm{~m}, \mathrm{P}=0.02$ ); (B) PFS (high VEGF group vs. control group: 5.8 vs. $12.1 \mathrm{~m}, \mathrm{P}=0.4$ ); (C) OS (high VEGF group vs. control group: 12.8 vs. $18.4 \mathrm{~m}, \mathrm{P}=0.08)$. VEGF, vascular endothelial growth factor; PFS, progression-free survival; iPFS, intracranial PFS; OS, overall survival.

PASSPORT study showed no grade 2 or more intracerebral hemorrhage occurred in brain metastasis patients treated with bevacizumab (28). A meta-analysis, including 8 clinical trials involving 8,713 patients with brain metastasis, found that the incidence of intracerebral hemorrhage was less than $1 \%$ after bevacizumab treatment (29). In the present study, more than $1 / 3$ patients received more than 10 cycles of bevacizumab treatment. Most treatment-related adverse events were leukopenia, fatigue, thrombocytopenia, anemia, et al. Most adverse events were manageable, and no new safety signals were observed. Only one patient discontinued bevacizumab treatment because of increasing albuminuria
( $>2 \mathrm{~g} / 24 \mathrm{~h})$, and this patient had albuminuria $(<2 \mathrm{~g} / 24 \mathrm{~h})$ before treatment.

Angiogenesis is thought to be associated with brain metastasis. VEGF is one of the most important factors to regulate angiogenesis, and play a role in recurrence, metastasis and death of patients with lung adenocarcinoma (24). Brain metastasis significantly reduce survival of lung adenocarcinoma patients, and symptoms of CNS caused by brain metastasis severely affect the quality of life. Therefore, it's crucial for patients with symptomatic brain metastases from lung adenocarcinoma to identify sensitive patients to antiangiogenic therapy through establishing effectively 
Table 4 Univariate analyses of iPFS

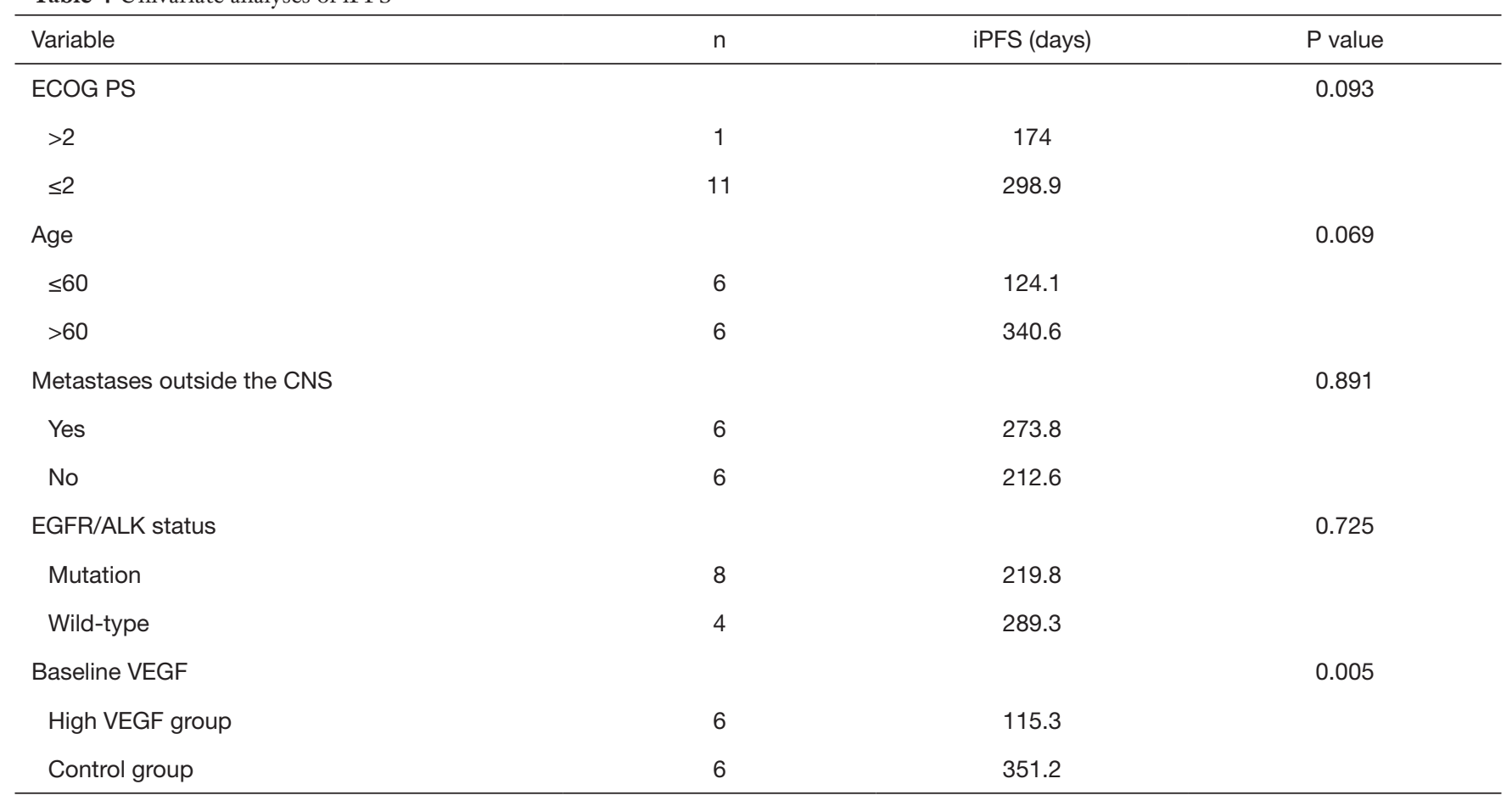

VEGF, vascular endothelial growth factor; CNS, central nervous system; EGFR, epidermal growth factor receptor; ALK, anaplastic lymphoma kinase; iPFS, intracranial progression-free survival.

Table 5 Multivariate analyses of iPFS

\begin{tabular}{lccllllr}
\hline Variable & $\mathrm{B}$ & $\mathrm{SE}$ & Wald & $\mathrm{df}$ & Sig. & $\operatorname{Exp}(\mathrm{B})$ & $95.0 \% \mathrm{Cl}$ for Exp (B) \\
\hline Baseline VEGF & 0.03 & 0.013 & 5.4 & 1 & 0.02 & 1.03 & $1.005-1.056$ \\
ECOG PS & -0.506 & 0.782 & 0.418 & 1 & 0.518 & 0.603 & $0.13-2.795$ \\
Age & -0.071 & 0.078 & 0.841 & 1 & 0.359 & 0.931 & $0.799-1.085$ \\
\hline
\end{tabular}

VEGF, vascular endothelial growth factor; iPFS, intracranial progression-free survival.

predictive biomarkers. The relationship between VEGF and efficacy of bevacizumab has remained ambiguous (30,31). Our results showed baseline serum VEGF was significantly associated with iPFS, indicating that VEGF could serve as a promising predictive biomarker.

This analysis is a retrospective, single-center clinical trial. Small sample size is the biggest limitation. So our conclusion need to be verified in randomized, multicenter trials. However, this is the first study to investigate the efficacy and safety of bevacizumab combined with chemotherapy in patients with symptomatic brain metastases from lung adenocarcinoma in China, and we provided a novel treatment choice for these patients. Furthermore, VEGF level, demonstrated to be a potential biomarker for bevacizumab treatment in this study, is convenient to be detected and has a promising clinical application value.

In conclusion, our results suggest that bevacizumab combination with chemotherapy is effective and safe, and provides a new therapeutic option for the patients with symptomatic brain metastases. The baseline serum VEGF could serve as a promising biomarker to predict the efficacy of bevacizumab combined with chemotherapy in symptomatic brain metastases from lung adenocarcinoma.

\section{Acknowledgments}

Funding: This research was supported by Shanghai Municipal Natural Science Foundation (16ZR1404300) and 
Scientific Research Foundation Huashan Hospital [787].

\section{Footnote}

Conflicts of Interest: The authors have no conflicts of interest to declare.

Ethical Statement: The authors are accountable for all aspects of the work in ensuring that questions related to the accuracy or integrity of any part of the work are appropriately investigated and resolved. The protocol was approved by the Institutional Review Board Committee of Huashan Hospital, Shanghai, China (No. KY2017-010).

\section{References}

1. Villano JL, Durbin EB, Normandeau C, et al. Incidence of brain metastasis at initial presentation of lung cancer. Neuro Oncol 2015;17:122-8.

2. Lin YJ, Chiu HY, Chiou MJ, et al. Trends in the incidence of primary malignant brain tumors in Taiwan and correlation with comorbidities: A population-based study. Clin Neurol Neurosurg 2017;159:72-82.

3. Bulbul A, Forde PM, Murtuza A, et al. Systemic Treatment Options for Brain Metastases from Non-Small-Cell Lung Cancer. Oncology (Williston Park) 2018;32:156-63.

4. Russo AE, Priolo D, Antonelli G, et al. Bevacizumab in the treatment of NSCLC: patient selection and perspectives. Lung Cancer (Auckl) 2017;8:259-69.

5. Sandler A, Gray R, Perry MC, et al. Paclitaxel-carboplatin alone or with bevacizumab for non-small-cell lung cancer. N Engl J Med 2006;355:2542-50.

6. Reck M, von Pawel J, Zatloukal P, et al. Phase III trial of cisplatin plus gemcitabine with either placebo or bevacizumab as first-line therapy for nonsquamous non-small-cell lung cancer: AVAil. J Clin Oncol 2009;27:1227-34.

7. Zustovich F, Ferro A, Lombardi G, et al. BevacizumabBased Therapy for Patients with Brain Metastases from Non-Small-Cell Lung Cancer: Preliminary Results. Chemotherapy 2014;60:294-9.

8. Chikaishi Y, Kanayama M, Taira A, et al. Effect of erlotinib plus bevacizumab on brain metastases in patients with non-small cell lung cancer. Ann Transl Med 2018;6:401.

9. Stefanou D, Stamatopoulou S, Sakellaropoulou A, et al. Bevacizumab, pemetrexed and carboplatin in first-line treatment of non-small cell lung cancer patients: Focus on patients with brain metastases. Oncol Lett 2016;12:4635-42.
10. Besse B, Le Moulec S, Mazieres J, et al. Bevacizumab in Patients with Nonsquamous Non-Small Cell Lung Cancer and Asymptomatic, Untreated Brain Metastases (BRAIN): A Nonrandomized, Phase II Study. Clin Cancer Res 2015;21:1896-903.

11. Bennouna J, Falchero L, Schott R, et al. Bevacizumab in Combination with Platinum-Based Chemotherapy in Patients with Advanced Non-Squamous Non-Small Cell Lung Cancer with or without Brain Metastases: A French Cohort Study (EOLE). Oncology 2018;94:55-64.

12. Vredenburgh JJ, Cloughesy T, Samant $M$, et al. Corticosteroid use in patients with glioblastoma at first or second relapse treated with bevacizumab in the BRAIN study. Oncologist 2010;15:1329-34.

13. Wick W, Gorlia T, Bendszus M, et al. Lomustine and Bevacizumab in Progressive Glioblastoma. N Engl J Med 2017;377:1954-63.

14. Berghoff AS, Sax C, Klein M, et al. Alleviation of brain edema and restoration of functional independence by bevacizumab in brain-metastatic breast cancer: a case report. Breast Care (Basel) 2014;9:134-6.

15. Liang X, Huang R, Zhan Q. Shanghai expert consensus on the management of brain metastasis of non-small cell lung cancer with driver gene mutations. China Oncology 2019;29:10.

16. Nishino M, Jackman DM, Hatabu H, et al. New Response Evaluation Criteria in Solid Tumors (RECIST) guidelines for advanced non-small cell lung cancer: comparison with original RECIST and impact on assessment of tumor response to targeted therapy. AJR Am J Roentgenol 2010;195:W221-8.

17. Alexander BM, Brown PD, Ahluwalia MS, et al. Clinical trial design for local therapies for brain metastases: a guideline by the Response Assessment in NeuroOncology Brain Metastases working group. Lancet Oncol 2018;19:e33-42.

18. Wen PY, Chang SM, Van den Bent MJ, et al. Response Assessment in Neuro-Oncology Clinical Trials. J Clin Oncol 2017;35:2439-49.

19. Chen AP, Setser A, Anadkat MJ, et al. Grading dermatologic adverse events of cancer treatments: the Common Terminology Criteria for Adverse Events Version 4.0. J Am Acad Dermatol 2012;67:1025-39.

20. Naikoo NA, Dil A, Rasool R, et al. Upregulation of vascular endothelial growth factor (VEGF), its role in progression and prognosis of non-small cell lung carcinoma. Cancer Genet 2017;216-217:67-73.

21. Villaruz LC, Socinski MA. The role of anti-angiogenesis 
in non-small-cell lung cancer: an update. Curr Oncol Rep 2015;17:26.

22. Fischer I, Cunliffe CH, Bollo RJ, et al. High-grade glioma before and after treatment with radiation and Avastin: initial observations. Neuro Oncol 2008;10:700-8.

23. Ilhan-Mutlu A, Osswald M, Liao Y, et al. Bevacizumab Prevents Brain Metastases Formation in Lung Adenocarcinoma. Mol Cancer Ther 2016;15:702-10.

24. Fu Y, Hu J, Du N, et al. Bevacizumab plus chemotherapy versus chemotherapy alone for preventing brain metastasis derived from advanced lung cancer. J Chemother 2016;28:218-24.

25. De Bock K, Cauwenberghs S, Carmeliet P. Vessel abnormalization: another hallmark of cancer? Molecular mechanisms and therapeutic implications. Curr Opin Genet Dev 2011;21:73-9.

26. Friedman HS, Prados MD, Wen PY, et al. Bevacizumab alone and in combination with irinotecan in recurrent

Cite this article as: Zhan Q, Miao F, Huang R, Zhou X, Ge $M$, Liang X. Efficacy and safety of bevacizumab combined with chemotherapy in symptomatic brain metastases from lung adenocarcinoma: a retrospective analysis. J Thorac Dis 2019;11(11):4725-4734. doi: 10.21037/jtd.2019.10.49 glioblastoma. J Clin Oncol 2009;27:4733-40.

27. De Braganca KC, Janjigian YY, Azzoli CG, et al. Efficacy and safety of bevacizumab in active brain metastases from non-small cell lung cancer. J Neurooncol 2010;100:443-7.

28. Socinski MA, Langer CJ, Huang JE, et al. Safety of bevacizumab in patients with non-small-cell lung cancer and brain metastases. J Clin Oncol 2009;27:5255-61.

29. Yang L, Chen CJ, Guo XL, et al. Bevacizumab and risk of intracranial hemorrhage in patients with brain metastases: a meta-analysis. J Neurooncol 2018;137:49-56.

30. Hanibuchi M, Kim SJ, Fidler IJ, et al. The molecular biology of lung cancer brain metastasis: an overview of current comprehensions and future perspectives. J Med Invest 2014;61:241-53.

31. Guo S, Martin MG, Tian C, et al. Evaluation of Detection Methods and Values of Circulating Vascular Endothelial Growth Factor in Lung Cancer. J Cancer 2018;9:1287-300. 
Table S1 Detailed clinical characteristics

\begin{tabular}{|c|c|c|c|c|c|c|c|c|c|c|c|c|c|c|c|c|}
\hline No. & Sex & Age & PS & $\begin{array}{l}\text { Extra-CNS } \\
\text { metastases }\end{array}$ & $\begin{array}{l}\text { Mum of } \\
\text { BMs }\end{array}$ & $\begin{array}{l}\text { Driver- } \\
\text { oncogene }\end{array}$ & $\begin{array}{c}\text { Therapy before } \\
\text { BEV }\end{array}$ & Chemotherapy & Efficacy & $\begin{array}{l}\text { Efficacy } \\
\text { of CNS }\end{array}$ & Symptom of CNS & $\begin{array}{l}\text { Improve } \\
\text { of Sym }\end{array}$ & $\begin{array}{c}\text { Num of } \\
\text { Bev }\end{array}$ & $\begin{array}{l}\text { Cause of } \\
\text { withdrawal }\end{array}$ & Site of PD & $\begin{array}{c}\text { Therapy after } \\
\text { withdrawal }\end{array}$ \\
\hline 1 & $\mathrm{~F}$ & 49 & 3 & Bone & Multiple & $\begin{array}{l}\text { EGFR } 21 \\
\text { Exon L858r }\end{array}$ & $\begin{array}{l}\text { Erlotinib, } \\
\text { osimertinib }\end{array}$ & $P$ & SD & SD & Limb weakness, dizzy & Yes & 6 & AE & N/A & BSC \\
\hline 2 & $\mathrm{~F}$ & 70 & 1 & None & Single & None & SRS & $\mathrm{PP}$ & PR & $\mathrm{PR}$ & Left limb weakness & Yes & 12 & PD & Lung, PBM(N) & BSC \\
\hline 3 & M & 52 & 1 & None & Multiple & EGFR 19del & $\begin{array}{c}\text { WBRT, } \\
\text { chemotherapy, } \\
\text { Gefitinib }\end{array}$ & TC & SD & SD & Headache & Yes & 17 & PD & LM & V-P shunt \\
\hline 4 & M & 71 & 1 & Lymph node & Multiple & None & SRS & PP & $\mathrm{N} / \mathrm{A}$ & $\mathrm{N} / \mathrm{A}$ & N/A & N/A & 1 & ED & $\mathrm{N} / \mathrm{A}$ & BSC \\
\hline 5 & M & 61 & 2 & None & Multiple & None & N/A & $\mathrm{PP}$ & N/A & N/A & $\mathrm{N} / \mathrm{A}$ & $\mathrm{N} / \mathrm{A}$ & 1 & AE & N/A & BSC \\
\hline 6 & M & 49 & 1 & None & Multiple & None & PC & TC & SD & SD & $\begin{array}{l}\text { Limb weakness, foot } \\
\text { numbness, dysuresia }\end{array}$ & Yes & 5 & PD & LM & BSC \\
\hline 7 & M & 69 & 1 & $\begin{array}{l}\text { Adrenal gland, } \\
\text { lymph node }\end{array}$ & Multiple & EGFR 19del & $\begin{array}{l}\text { chemotherapy, } \\
\text { icotinib }\end{array}$ & TC & PD & PD & Limb weakness & No & 2 & PD & $\begin{array}{c}\text { Lung, } \\
\text { PBM(N+E) }\end{array}$ & $\begin{array}{l}\text { Osimertinib, } \\
\text { SRS }\end{array}$ \\
\hline 8 & $\mathrm{~F}$ & 42 & 2 & $\begin{array}{l}\text { Lung, } \\
\text { pericardium, } \\
\text { bone }\end{array}$ & Multiple & EGFR 19del & Gefitinib & D & PD & PD & Headache, dizzy & No & 1 & PD & LM, PBM(N) & $\begin{array}{l}\text { Osimertinib, } \\
\text { SRS }\end{array}$ \\
\hline 9 & $\mathrm{~F}$ & 60 & 1 & None & Multiple & $\begin{array}{l}\text { EGFR } 21 \\
\text { Exon L858r }\end{array}$ & $\begin{array}{l}\text { WBRT + SRS, } \\
\text { erlotinib, } \\
\text { osimertinib }\end{array}$ & $\mathrm{PP}$ & SD & SD & Dysphasia & Yes & 15 & PD & Liver & BSC \\
\hline 10 & M & 61 & 2 & None & Multiple & None & N/A & PP & SD & SD & $\begin{array}{c}\text { Memory loss, } \\
\text { dysphasia, reading \& } \\
\text { writing difficulties }\end{array}$ & Yes & 3 & PD & Lung & GP, BSC \\
\hline 11 & M & 71 & 1 & None & multiple & None & SRS & PP & PR & $\mathrm{PR}$ & $\begin{array}{l}\text { Dysphasia, hearing } \\
\text { loss, slow reaction }\end{array}$ & Yes & 19 & N/A & $\mathrm{N} / \mathrm{A}$ & $\mathrm{N} / \mathrm{A}$ \\
\hline 12 & M & 64 & 2 & Lung & multiple & ALK fusion & Crizotinib & PP & SD & PR & Headache, dizzy & Yes & 4 & PD & Lung & BSC \\
\hline 13 & M & 52 & 1 & Liver, bone & single & $\begin{array}{l}\text { EGFR } 20 \\
\text { Exon T790m }\end{array}$ & $\begin{array}{l}\text { Surgery, } \\
\text { osimertinib }\end{array}$ & PP & PR & PR & Headache & Yes & 15 & N/A & $\mathrm{N} / \mathrm{A}$ & N/A \\
\hline 14 & M & 59 & 2 & Lung, pleura & N/A & EGFR 19del & $\mathrm{N} / \mathrm{A}$ & D & PD & PD & $\begin{array}{l}\text { Headache, } \\
\text { unconsciousness }\end{array}$ & No & 1 & PD & LM & $\begin{array}{l}\text { Icotinib, } \\
\text { osimertinib }\end{array}$ \\
\hline
\end{tabular}

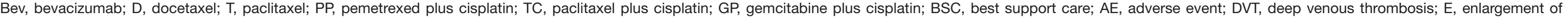

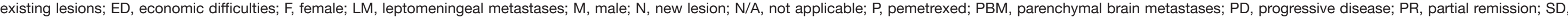
stable disease; SRS, stereotactic radiosurgery; V-P shunt, ventriculoperitoneal shunt; WBRT, whole brain irradiation. 